\title{
VARIANTE ANATÓMICA DE LA ARTERIA CÓLICA MEDIA QUE DA ORIGEN A LA ARTERIA CÓLICA IZQUIERDA
}

\author{
Victoria García Ríos, Emilia Cerchiari
}

\author{
Departamento de Anatomía, Universidad de la República, Montevideo, Uruguay
}

\begin{abstract}
RESUMEN
Introducción: basado en la embriología pueden diferenciarse el colon derecho y colon izquierdo según su vascularización provenga de las arterias mesentérica superior o inferior respectivamente. La descripción clásica de la arteria cólica media, originada en la arteria mesentérica superior, supone que la misma vasculariza el colon transverso conjuntamente con la arteria cólica izquierda que tiene origen en la arteria mesentérica inferior. Reporte de caso: se reporta el caso de una variante de la arteria cólica media que incide en la vascularización del colon izquierdo. La misma fue hallada durante la disección de un cadáver de sexo femenino, fijado en solución de formaldehído, donado al Departamento de Anatomía de la Facultad de Medicina de la Universidad de la República. Se identificó a la arteria cólica media en su origen habitual, presentando una variante del trayecto según la cual descendió por el flanco izquierdo de la aorta abdominal, originando a la arteria cólica izquierda. Distalmente alcanzó el recto para participar de su vascularización. Discusión: las variantes en la vascularización del colon izquierdo son menos frecuentes respecto a las del colon derecho. En este caso una rama de la arteria mesentérica superior, incide en la vascularización del colon izquierdo. Esta variante no sólo plantea potenciales complicaciones a la hora del abordaje, sino que plantea la duda de cuál sería una resección oncológica adecuada para un tumor del colon izquierdo. El conocimiento anatómico de la vascularización del colon y sus variantes, sigue vigente y tiene implicancias en la práctica clínica quirúrgica.
\end{abstract}

Palabras claves: variante vascular; colon izquierdo; arteria mesentérica superior; arteria mesentérica inferior

\begin{abstract}
Introduction: based on embryology right and left colon can be distinguished according to whether they are vascularized by the superior or inferior mesenteric artery respectively. In its classic description, middle colic artery arises from the superior mesenteric artery and supplies the transverse colon. Left colic artery usually originates from the inferior mesenteric artery. Case report: we described an anatomical variation in which middle colic artery directly irrigates de left colon. It was found during the dissection of a female cadaver fixed in formalin solution, in the Medicine Faculty of the University of the Republic in Montevideo, Uruguay. Middle colic artery was identified from its usual origin, and had a variation of its course, descending at the left flank of the abdominal aorta, and giving rise to the left colic artery. Distally it participated in the rectum supply. Discussion: anatomical variations of the left colon vascularization has been said to be less frequent than those of the right colon. In this case a branch of the superior mesenteric artery supplied the left colon. These could not only implicate possible complications during colon surgery, but also set an interrogant about the best oncologic solution in cases of left colon tumor. Importance of anatomical knowledge of left colon vascularization and its variations remains important and has implicances in surgical practice.
\end{abstract}

Key words: vascular variant; left colon; superior mesenteric artery; inferior mesenteric artery

\begin{tabular}{lllll}
\hline * Correspondencia & a: & Victoria & García & Ríos. \\
gariosmv@gmail.com
\end{tabular}

Recibido: 13 de Enero, 2020. Revisado: 6 de Febrero, 2020. Aceptado: 16 de Febrero, 2020. 


\section{INTRODUCCIÓN}

Clásicamente se ha descrito que las tres ramas que parten de la cara anterior y antero-lateral izquierda de la aorta abdominal (AA) irrigan al aparato digestivo infradiafragmático.

El tronco celíaco (TC) es la arteria encargada de la vascularización del piso supramesocólico y se acepta que la arteria mesentérica superior (AMS) en conjunto con la arteria mesentérica inferior (AMI) se encargan de la irrigación del piso inframesocólico.

La AMS irriga, el intestino delgado hasta el sector distal por sus ramas yeyuno-ileales, y el sector del colon que por su origen embrionario denominamos "colon derecho": ciego, apéndice y colon ascendente (por intermedio de las arterias ileo-cólica y cólica derecha), y los dos tercios derechos del colon transverso (por la rama derecha de la arteria cólica media (ACM)).

Por otra parte, el territorio vascular de la AMI abarca: el tercio distal del colon transverso, ángulo cólico izquierdo, colon descendente, sigmoide y recto. Estos están vascularizados respectivamente por las ramas ascendente y descendente de la arteria cólica izquierda ( $\mathrm{ACl}$ ), las arterias sigmoideas, y la arteria rectal superior, que constituye la rama terminal de la $\mathrm{AMI}$. Entendemos por ACI como la primera rama de la AMI que se dirige al ángulo cólico izquierdo (Latarjet y Ruiz Liard, 2004; Rouviere y Delmas, 2005; Szereszwski, 2009).

La anastomosis intermesentérica en el límite entre los dos tercios proximales y el tercio distal del colon trasverso, próximo al borde mesial del órgano y todo a lo largo de este, forma una arcada marginal bien descrita por Drummond (1913).

Esta distribución es producto de los fenómenos de crecimiento, rotación y fusión del tubo digestivo primitivo y la vasculogénesis durante el desarrollo embrionario. Una vez cerrado, el tubo digestivo primitivo queda suspendido por un mesenterio dorsal por donde discurren los vasos del saco vitelino, provenientes de la aorta dorsal. Con el crecimiento del embrión, varios de estos vasos se fusionan y otros desaparecen. Persisten tres arterias que transitan en los mesos hacia el tubo digestivo: en la región gastrohepática el TC; en la región del asa vitelina la AMS, y en la región del intestino posterior la AMI. Al mismo tiempo que se alarga, el asa vitelina inicia un proceso de rotación y elongación en pos de un eje constituido por la AMS, permitiendo establecer una porción previtelina y una posvitelina. La región previtelina se ubica a la derecha de la línea media y la posvitelina a la izquierda, determinando en el adulto la disposición definitiva y vascularización del tubo digestivo (Moore, 2008; Flores, 2015; Sadler, 2016). Determinarán también el concepto anatomo-quirúrgico de colon derecho y colon izquierdo según su irrigación sea dada por la AMS o AMI respectivamente.

El objetivo del siguiente trabajo es reportar el caso de una variante en el origen de la $\mathrm{ACl}$, y en el trayecto y distribución de la ACM, la cual incide directamente en la vascularización del colon izquierdo.

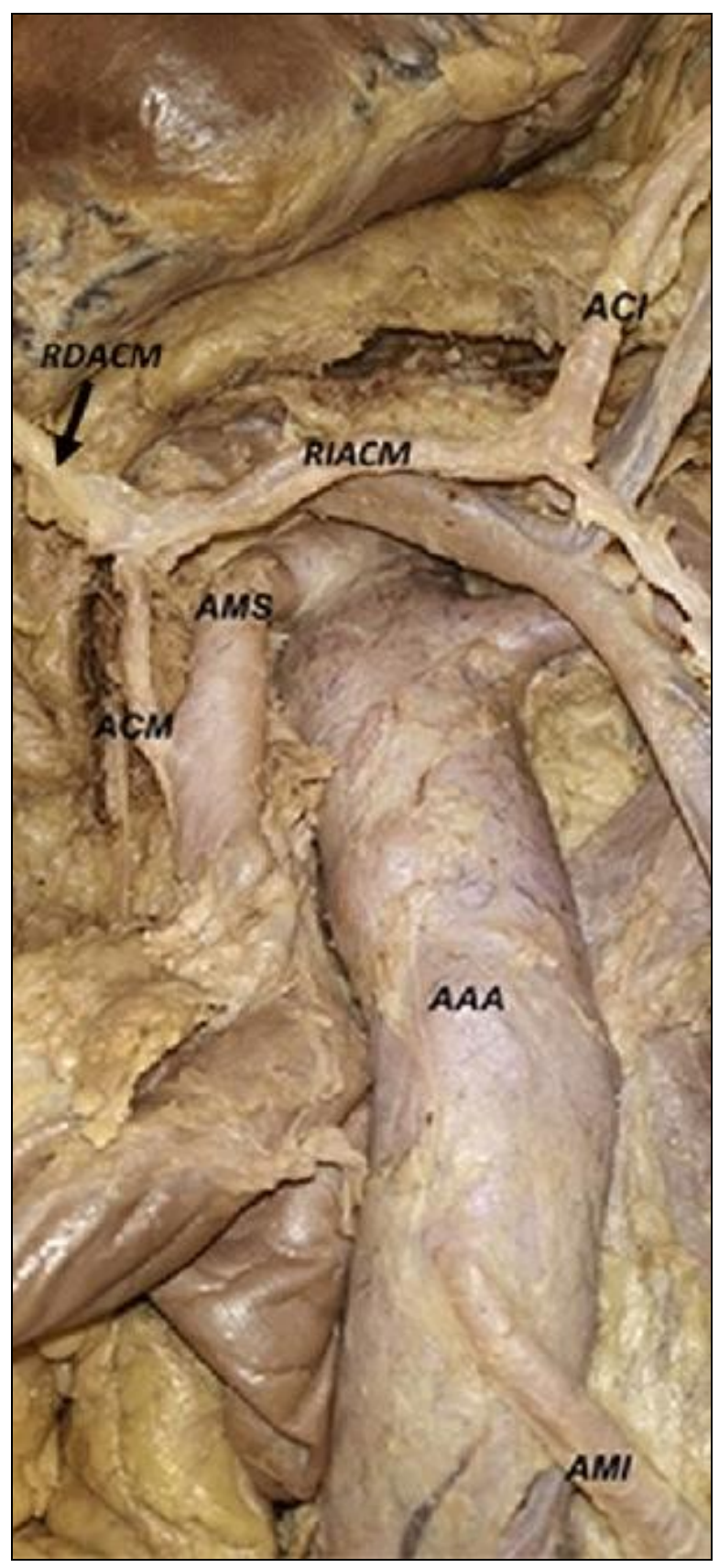

Figura 1: origen de la ACM. AA: aorta abdominal; AMS: arteria mesentérica superior; $\mathrm{ACM}$ : arteria cólica media; RDACM: rama derecha de la arteria cólica media; RIACM: rama izquierda de la arteria cólica media; $\mathrm{ACI}$ : arteria cólica izquierda; AMI: arteria mesentérica inferior. 


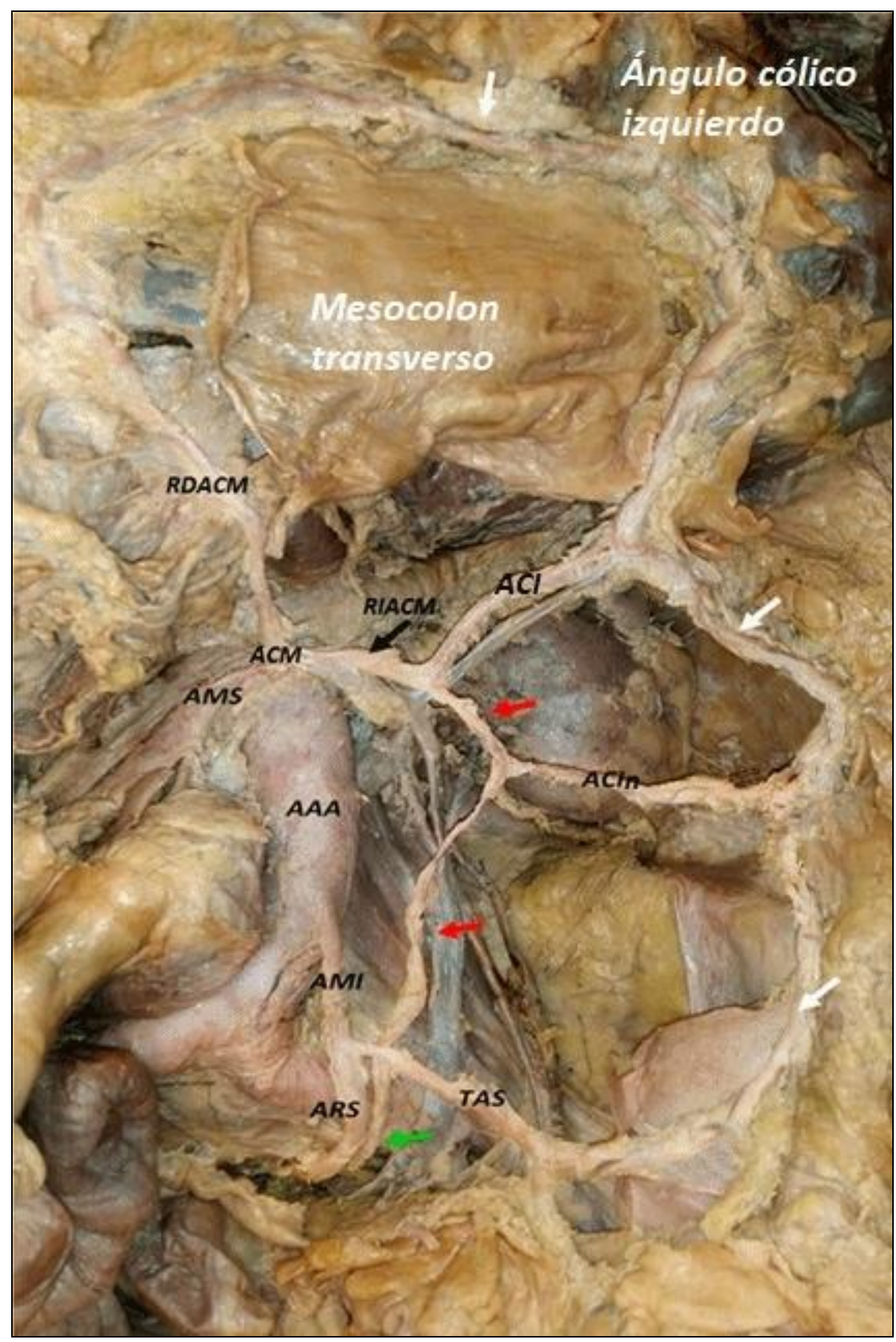

Figura 2: Disección transperitoneal de la aorta abdominal y mesocolon izquierdo. AA: aorta abdominal; AMS: arteria mesentérica superior; ACM: arteria cólica media; RDACM: rama derecha de la arteria cólica media; RIACM: rama izquierda de la arteria cólica media; ACI: arteria cólica izquierda; ACIn: arteria cólica intermedia; AMI: arteria mesentérica inferior; ARS: arteria rectal superior; TAS: tronco de arterias sigmoideas. Flechas rojas: arco vascular accesorio. Flechas blancas: arco marginal del colon. Flecha verde: rama terminal para el recto.

\section{REPORTE DEL CASO}

Reportamos el hallazgo de una variante anatómica en la distribución de la ACM durante la disección abdominal de un cadáver de sexo femenino de raza blanca, fijado en solución en base a formol. El mismo fue donado al Departamento de Anatomía de la Facultad de Medicina de la Universidad de la República en Montevideo, Uruguay. 
Se accedió a la cavidad abdominal por una incisión mediana xifo-púbica, y otra perpendicular a ésta, a nivel de la cicatriz umbilical. Se realizó un abordaje transperitoneal de la AA y sus ramas viscerales.

Se identificó a la ACM en su origen habitual a partir de la AMS (figura 1), presentando una variante anatómica, según la cual, su rama izquierda dio origen a la $\mathrm{ACl}$, la misma se distribuyó al ángulo cólico izquierdo y al colon descendente. En su trayecto (figura 2), configuró un segundo arco vascular (flechas rojas), paralelo al arco marginal del colon (flechas blancas), donde originó como segunda rama una arteria cólica intermedia que colaboró con la irrigación del colon descendente. Distalmente transcurrió paralela a la arteria rectal superior, llegando hasta el recto para participar en su vascularización (flecha verde).

No encontramos otras variantes de las ramas viscerales de la AA.

\section{DISCUSIÓN}

En términos generales, las variantes vasculares que involucran al colon izquierdo son menos frecuentes respecto a las del colon derecho (Manyama et al., 2019; Yoo et al., 2011).Nelson et al (1987) en su estudio sobre las variantes anatómicas de las arterias que irrigan al intestino infradiafragmático, reportan la distribución anatómica clásica solamente en el $24 \%$ de los casos para el TC, en el $22 \%$ para la AMS, y en un $16 \%$ para la $\mathrm{AMI}$, donde la variante más frecuente es un tronco común para el origen de la $\mathrm{ACl}$ y una arteria sigmoidea.

En cuanto a la $\mathrm{ACl}$, su definición ha variado en distintos trabajos: Vandamme y Van Der Schuren (1976) la define como la arteria originada en la AMl que se dirige al ángulo cólico izquierdo, mientras que Horton y Fishman (2002) la define como la arteria que se dirige al ángulo cólico izquierdo independientemente de su origen (estando ausente hasta en un $12 \%$ de los casos). Teniendo en cuenta estas definiciones, interpretamos lo hallado como una $\mathrm{ACl}$ cuyo origen se da en la ACM. En cuanto a la frecuencia de este hallazgo, Vandamme y Van Der Schuren (1976) reporta la existencia en el $7 \%$ de los casos, de una arteria originada en la AMS o una de sus ramas, que se dirige al ángulo cólico izquierdo a la cual denominan arteria cólica izquierda accesoria. Con similar implicancia, Yoo et al. (2011) reportan un caso en el cual la AMI se origina de la AMS para luego mantener una ramificación anatómica clásica. Registros menos frecuentes han sido descritos por autores como
Kachlika et al. (2009) y Manyama et al. (2019) en los cuales la $\mathrm{ACl}$ nace de una arteria mesentérica media o incluso directamente de la AA.

El hallazgo presentado, reviste importancia no sólo anatómica, sino también clínico-quirúrgica, e implica una variante en el territorio vascular de la AMS. Tiene directa implicancia frente a un abordaje quirúrgico, y fundamentalmente en el contexto de la cirugía oncológica, en donde la resección linfática determina el nivel de ligadura vascular, y esta última la resección visceral. (Kobayashi et al., 2006; Minetti y Manoni, 2013). Respecto a la distribución de las arterias que irrigan al colon izquierdo, Bruzzi et al. (2018) concluye que el arco marginal del colon, es la arteria paralela al colon que suministra la vasa recta, similar a las descripciones clásicas ya referidas. Según este autor, la principal conexión intermesentérica se topografía a nivel del ángulo cólico izquierdo, y su prevalencia es del $100 \%$.A diferencia de esto, en el caso presentado el arco marginal mayoritariamente se conforma por ramas de la AMS, siendo la conexión intermesentérica topografiada más distal en el colon. Debemos concluir que el estudio de las arterias que irrigan el colon tiene gran significancia clínico y quirúrgica, siendo clave para la planificación preoperatoria y la disminución de complicaciones intraoperatorias.

\section{Conflicto de interés}

Los autores declaran no tener conflicto de interés.

\section{Financiamiento}

Los autores declaran no haber recibido ningún tipo de financiación económica por parte de terceros.

\section{Aprobación ética}

El material proviene de cadáveres donados a voluntad personal, a la facultad de Medicina de la Universidad de la República, Montevideo, Uruguay.

\section{Consentimiento informado}

El Departamento de Anatomía de la Facultad de Medicina de la Universidad de la República, Montevideo, Uruguay, cuenta con el consentímiento informado en vida de donantes cadavéricos para la docencia e investigación.

\section{Contribuciones}

VGR: disección cadavérica, escritura del manuscrito. EC: escritura y revisión del manuscrito. 


\section{AGRADECIMIENTOS}

A quienes en vida deciden donar su cuerpo a nuestra facultad para la docencia e investigación de la Anatomía.

\section{BIBLIOGRAFÍA}

Bruzzi M, M'harzi L, El Batti S, AbouGhazaleh R, Taieb J, Poghosyan T, Berger A, Chevallier J M, Douard R. 2018. Inter-mesenteric connections between the superior and inferior mesenteric arteries for left colonic vascularization: implications for colorectal surgery; SurgRadiol Anat. 41: 255-64.

Drummond H. 1913. The arterial supply of the rectum and pelvic colon. The British J. of Surg. 1: 677-85.

Flores V. 2015. Bases moleculares y celulares de la histogénesis, la morfogénesis y las alteraciones del desarrollo. Orientada a la formación médica. Embriología Humana. Buenos Aires Argentina. Editorial Panamericana. 174-82.

Horton K, Fishman E. 2002. Volume-rendered 3D CT of the Mesenteric Vasculature: Normal Anatomy, Anatomic Variants, and Pathologic Conditions. Radiographics. 22: 161-72.

Yoo SJ, Ku MJ, Cho SS, Yoon SP. 2011. A Case of the Inferior Mesenteric Artery Arising from the Superior Mesenteric Artery in a Korean Woman. J Korean Med Sci. 26: 1382-85.

Kachlik D, Laco J, Turyna R, Baca V. 2009. A very rare variant in the colon supply - Arteria mesentérica media. Biomed Pap Med Fac Univ Palacky Olomouc Czech Repub. 153: 79-82.

Kobayashi M, Morishita S, Okabayashi T, Miyatake K, Okamoto K, Namikawa T, Ogawa
Y, Araki K. 2006. Preoperative assessment of vascular anatomy of inferior mesenteric artery by volume-rendered 3D-CT for laparoscopic lymph node dissection with left colic artery preservation in lower sigmoid and rectal cancer. World J Gastroenterol. 12: 553-55.

Latarjet M, Ruiz Liard A. 2004. Intestino grueso. Anatomía Humana. $4^{\mathrm{a}}$ edición. Madrid España; Editorial Médica Panamericana. 1581633.

Manyama M, Malyango A, Raoof A, Mligiliche $N$, Msuya C, Nassir N. 2019. A variant source of arterial supply to the ascending, transverse and descending colon. Surg Radiol Anat. 41. 138386.

Minetti A, Manoni J. 2013. Capítulo V: Bases para el tratamiento quirúrgico del cáncer colorrectal; Rev Argent Coloproct. 24:118-26.

Moore L. 2008. Embriología clínica del desarrollo del ser humano; 8 a edición; Barcelona España; Editorial Elsevier. 266-75.

Nelson $T$, Pollak $R$, Jonasson $O$, Abcarian $H$. 1987. Anatomic Variants of the Celiac, Superior Mesenteric and Inferior Mesenteric Arteries and Their Clinical Relevance. Clinic. Anat. 1: 75-91.

Rouviere H, Delmas A. 2005. Anatomía Humana, Descriptiva, Topográfica y Funcional; 11 a edición. Barcelona - España. Editorial Masson. 400-60.

Sadler T.W. 2016. Embriología médica de Langman.13a edición. Editorial Lippincott Castellano. 239-49.

Szereszwski, J. 2009. Anatomía quirúrgica del colon; Cirugía Digestiva, F. Galindo III-300. 16.

Vandamme J, Van Der Schuren G. 1976. Reevaluation of the colic irrigation from the superior mesenteric artery. Acta. Anat. 95: 57888. 